\title{
Computed tomography and magnetic resonance imaging evaluation of pericardial disease
}

\author{
Edward T. D. Hoey ${ }^{1}$, Muhammad Shahid ${ }^{2}$, Richard W. Watkin ${ }^{2}$ \\ ${ }^{1}$ Department of Radiology, ${ }^{2}$ Department of Cardiology, Heart of England NHS Trust, Birmingham, UK \\ Correspondence to: Edward T. D. Hoey, MRCP, FRCR. Department of Radiology, Heartlands Hospital, Bordesley Green, Birmingham B9 5SS, UK. \\ Email: edwardhoey1@gmail.com.
}

\begin{abstract}
Pericardial diseases are commonly encountered in clinical practice and may present as an isolated process or in association with various systemic conditions. Traditionally transthoracic echocardiography (TTE) has been the method of choice for the evaluation of suspected pericardial disease but increasingly computed tomography (CT) and magnetic resonance imaging (MRI) are also being used as part of a rational multi-modality imaging approach tailored to the specific clinical scenario. This paper reviews the role of CT and MRI across the spectrum of pericardial diseases.
\end{abstract}

Keywords: Computed tomography (CT); magnetic resonance imaging (MRI); pericardium

Submitted Dec 15, 2015. Accepted for publication Jan 06, 2016.

doi: $10.21037 /$ qims.2016.01.03

View this article at: http://dx.doi.org/10.21037/qims.2016.01.03

\section{Introduction}

Pericardial diseases are commonly encountered in clinical practice and may present as an isolated process or in association with various systemic conditions. In some instances pericardial pathology will be straightforward to diagnose based on typical clinical features and electrocardiogram findings and imaging tests may not be required but many pericardial diseases may present with vague and non-specific symptoms resulting in considerable diagnostic dilemmas for which imaging can play a crucial role in helping establish the diagnosis and guiding appropriate therapeutic interventions. Traditionally transthoracic echocardiography (TTE) has been the method of choice for the evaluation of suspected pericardial disease but increasingly computed tomography (CT) and magnetic resonance imaging (MRI) are also being used as part of a rational multi-modality imaging approach tailored to the specific clinical scenario (1). This paper reviews the role of CT and MRI across the spectrum of pericardial diseases.

\section{Pericardial anatomy and function}

The pericardium is composed of two layers: the outer fibrous pericardium and the inner visceral pericardium creating the pericardial sac or cavity in between (1). The fibrous pericardium is more distensible than the visceral layer and both have a thickness of $0.5-1.0 \mathrm{~mm}$. Under normal physiological conditions the pericardial cavity contains $20-25 \mathrm{~mL}$ of serous fluid which is an ultrafiltrate of plasma produced by mesothelial cells lining the visceral layer and drains via the lymphatics and thoracic duct $(2,3)$. The pericardial sac envelops the heart, proximal ascending aorta and pulmonary trunk as well as a small portion of the pulmonary veins. Inferiorly it is anchored to the central tendon of the diaphragm and anteriorly to the sternum via the sternopericardial ligament (2). There are two major pericardial sinuses-the transverse sinus describes the recess separating common coverage of the aorta and pulmonary artery anteriorly from coverage of the vena cava and pulmonary veins posteriorly. The oblique sinus is located behind the left atrium (LA) where a portion of the left atrial wall is separated from the pericardial space (4).

The pericardium has several important anatomical functions including isolating the heart from the adjacent lungs and pleura to reduce the spread of infection, fixation of the heart position within the thoracic cavity and reduction of friction resistance by the presence of pericardial fluid (2-4). Physiologically the pericardical sac helps prevent 

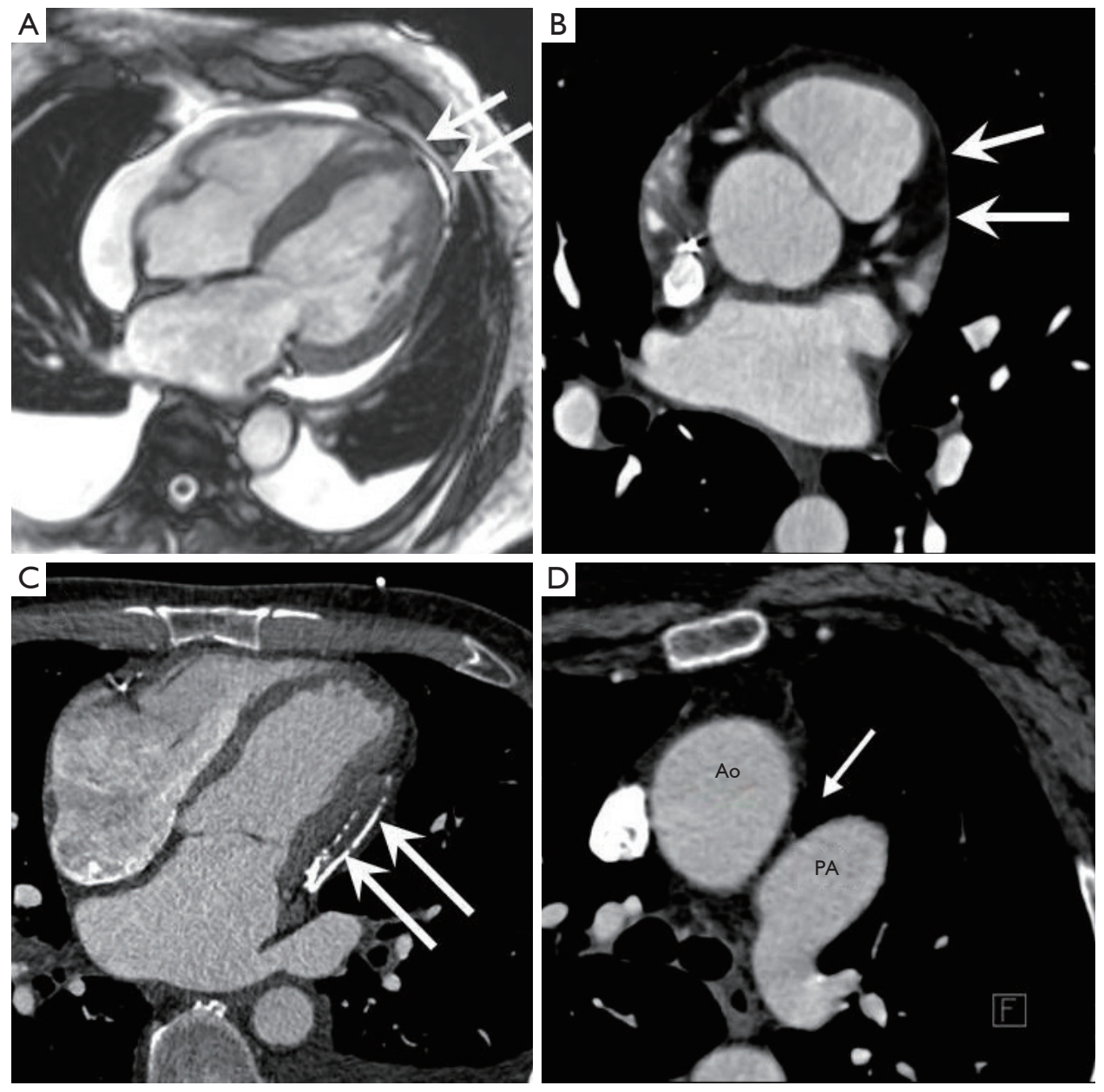

Figure 1 Examples of normal and abnormal pericardium. (A) Normal pericardial thickness (arrows) in a patient with a pericardial and pleural effusion secondary to cardiac amyloidosis; (B) normal pericardium on a CT coronary angiogram study (arrows); (C) pericardial calcific focus on a CT study in a patient with constrictive pericarditis (CP); (D) congenital absence of left sided pericardium on a CT study with interposition of lung tissue into the aorta-pulmonary artery recess (arrow). CT, computed tomography.

sudden dilatation of the cardiac chambers by exerting a restraining force, for example during exercise. It also helps to equalise compliance between the left ventricles (LV) and right ventricles (RV) (termed ventricular coupling) producing interdependence of filling. The pericardial sac has a small reserve volume and when exceeded acutely pressure is transmitted directly into the cardiac chambers. More chronic pressure elevation for example a slowing accumulating effusion may occur without constrictive physiology due to a more gradual shift in the pressure-volume relationship $(1,2)$.

\section{Multi-modality imaging techniques}

TTE is the initial imaging technique of choice for the majority of patients with suspected pericardial disease, but in many cases, TTE alone is insufficient to establish the diagnosis. The use of CT and/or MRI is very dependent on the specific clinical scenario mandating a highly individualized approach as both techniques have advantages and limitations across a range of disease processes. Both CT and MRI provide excellent anatomic delineation of the pericardium from adjacent tissue and enable a precise measurement of pericardial thickness (1). Normal pericardium appears as a thin linear band less than $2 \mathrm{~mm}$ thick on CT images (5). With cardiac MRI normal pericardium appears as a thin band of low-signal intensity on both T1, T2 and steady state free precession (SSFP) sequences as a result of its mainly fibrous structure and lack of water content (Figure 1A). A pericardial thickness greater than 
Table 1 Summary of institutional MRI protocol for pericardial disease

\begin{tabular}{|c|c|c|c|}
\hline Sequence & Planes and coverage & Goal of sequence & Parameters \\
\hline $\begin{array}{l}\text { T1-weighted double inversion } \\
\text { recovery fast spin-echo }\end{array}$ & $\begin{array}{l}\text { Axial from thoracic inlet } \\
\text { to diaphragm }\end{array}$ & $\begin{array}{l}\text { Anatomical overview; } \\
\text { pericardial thickness }\end{array}$ & $\begin{array}{l}\text { TR: } 1,000 \text { msec; TE: } 35-40 \mathrm{msec} ; 6 \mathrm{~mm} \\
\text { slice thickness; matrix: } 256 \times 256\end{array}$ \\
\hline \multicolumn{4}{|l|}{ Optional } \\
\hline
\end{tabular}

MRI, magnetic resonance imaging; LV, left ventricle; SSFP, steady state free precession.

$4 \mathrm{~mm}$ with MRI is considered abnormal (1).

CT has a higher spatial resolution than MRI (0.4 vs. $1-2 \mathrm{~mm})$ and is much superior for depicting pericardial calcification (Figure 1B,C). A standard prospective ECGgated cardiac CT examination with coverage from carina to diaphragm using $70 \mathrm{~mL}$ of iodinated contrast material is usually adequate for the assessment of pericardial disease. CT is also a much faster examination with a full cardiac dataset typically acquired in a single breath hold which is a major advantage in patients with orthopnoea. Conversely MRI has a better temporal resolution than CT (30-50 vs. 80-160 ms) which enables detection of rapid haemodynamic processes such as a septal bounce or respirophasic variation in septal excursion (1). Late gadolinium imaging with MRI is an excellent means of revealing ongoing pericardial inflammation and tissue characterization with cardiac MRI is superior to both CT and echocardiography. In addition MRI can be used to definitively characterize the tissue composition of cystic lesions and to assess the perfusion of a pericardial mass. Sites of malignant disease usually demonstrate high signal intensity on T2-weighted imaging due to increased free water content and show late gadolinium enhancement due to delayed washout associated with expansion of the extracellular space (6). A summary of our institutional MRI protocols for evaluation of pericardial disease is provided in Table 1.

\section{Congenital absence of the pericardium}

Congenital pericardial defects are rare (prevalence $0.002 \%$ ) and postulated to be caused by premature atrophy of the left common cardinal vein in utero which results in loss of the blood supply to the pleuropericardial membrane, which develops into the pericardium in adult life (7). Partial defects are most often left sided and manifest as a focal deficiency with chamber indentation at its free margins. Patients may be completely asymptomatic but herniation through the defect can cause angina, syncope and rarely sudden death (8). Complete pericardial defects permit excessive cardiac mobility due to non-existent constraint. Most cases are asymptomatic but occasionally can present with chest pain which is thought secondary to great vessel torsion or development of pleural-myocardial adhesions (7).

Absence of pericardium manifests on CT and MRI as an inability to identify pericardium along the cardiac borders and pathognomonic of left sided defects is interposition of lung tissue into the aorta-pulmonary artery recess (Figure 1D). Rightward axis shift with decubitus positioning has been described with left sided absence (Figure 2) (9).

\section{Acute pericarditis}

Acute inflammation of the pericardial layers is most often viral in origin with Coxsackie viruses most often implicated. 

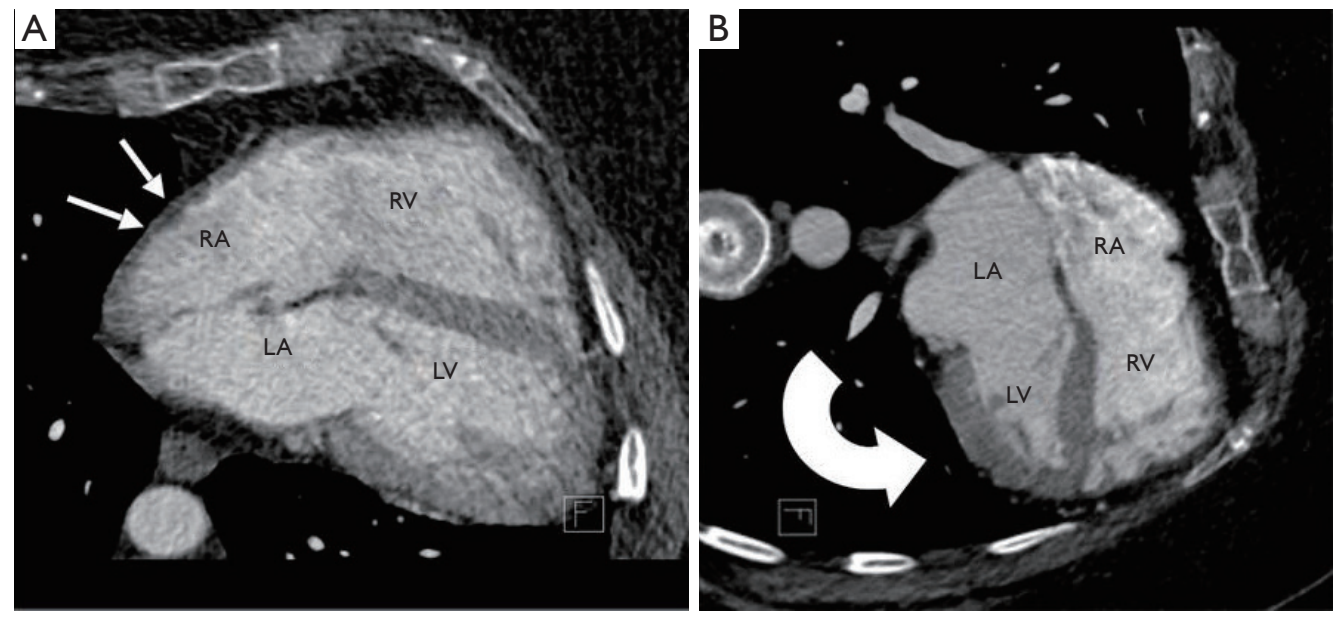

Figure 2 Congenital absence of left sided pericardium assessed with supine and decubitus CT studies. (A) Supine CT image showing pericardium over the RA only (arrows). There is pronounced leftward axis shift; (B) left lateral decubitus imaging shows rightward axis shift with elevation of the apex in keeping with a lack of pericardial restraint (curved arrow). LV, left ventricle; LA, left atrium; RV, right ventricle; RA, right atrium; CT, computed tomography.

Less common causes including post myocardial infarction syndrome (Dressler's syndrome), uraemia, tuberculosis, and neoplastic infiltration (1). Pericardial inflammation results in the formation of fresh highly vascularised granulation tissue accompanied by a variable amount of pericardial fluid accumulation. With acute uncomplicated pericarditis the diagnosis is usually established on the basis of typical clinical features and ECG findings with elevated systemic inflammatory markers. TTE may reveal a pericardial effusion but is frequently unremarkable and imaging is not usually required due to the self-limiting nature of the disease process (6). In general CT and/or MRI is usually reserved for those patients with atypical clinical features, those with a protracted disease course or with features suggesting evolution toward constrictive physiology (1).

With acute pericarditis, there is usually very minimal pericardial thickening and a pericardial effusion if present is small. Both CT and MRI can identify small or loculated pericardial effusions that might be missed with TTE. CT attenuation measurements enable characterization of pericardial fluid with a simple effusion having a density of 0-20 Hounsfield units. Attenuation greater than that of water suggests an exudate such as associated with malignancy, bacterial or tuberculous infection or haemopericardium. MRI can also provide non-invasive assessment of fluid composition. A simple pericardial effusion appears with low signal intensity on T1-weighted images and high signal intensity on T2-weighted images, including SSFP (6). A haemorrhagic component should be suspected with high signal intensity on T1-weighted images; likewise, haemorrhage or infection should be suspected when the effusion appears complex and inhomogeneous on SSFP (6). Late gadolinium enhancement may be seen and characteristically localizes along the pericardium and/or epi-myocardium (Figure 3). When an effusion is secondary to malignancy, an irregularly thickened pericardium or pericardial nodularity may be observed on both CT and MRI images (10).

Infrequently, acute pericarditis may be associated with larger volumes of rapidly accumulating fluid; once the limits of parietal pericardial stretch are exceeded, there is a rapid increase in intrapericardial pressure that compromises diastolic filling with resultant haemodynamic collapse (cardiac tamponade) (6). Urgent TTE with a view toward emergency pericardiocentesis is usually required and CT/ MRI is generally not indicated to avoid any treatment delay. Occasionally, a significant effusion may be initially detected on a CT or MRI study and the imaging specialist should be adept at spotting signs of impending tamponade, particularly diastolic collapse of the right-sided heart chambers which occurs when the intrapericardial pressure exceeds the intracavitary pressure.

\section{Constrictive pericarditis (CP)}

$\mathrm{CP}$ is characterized by fibrous or calcified thickening of the 

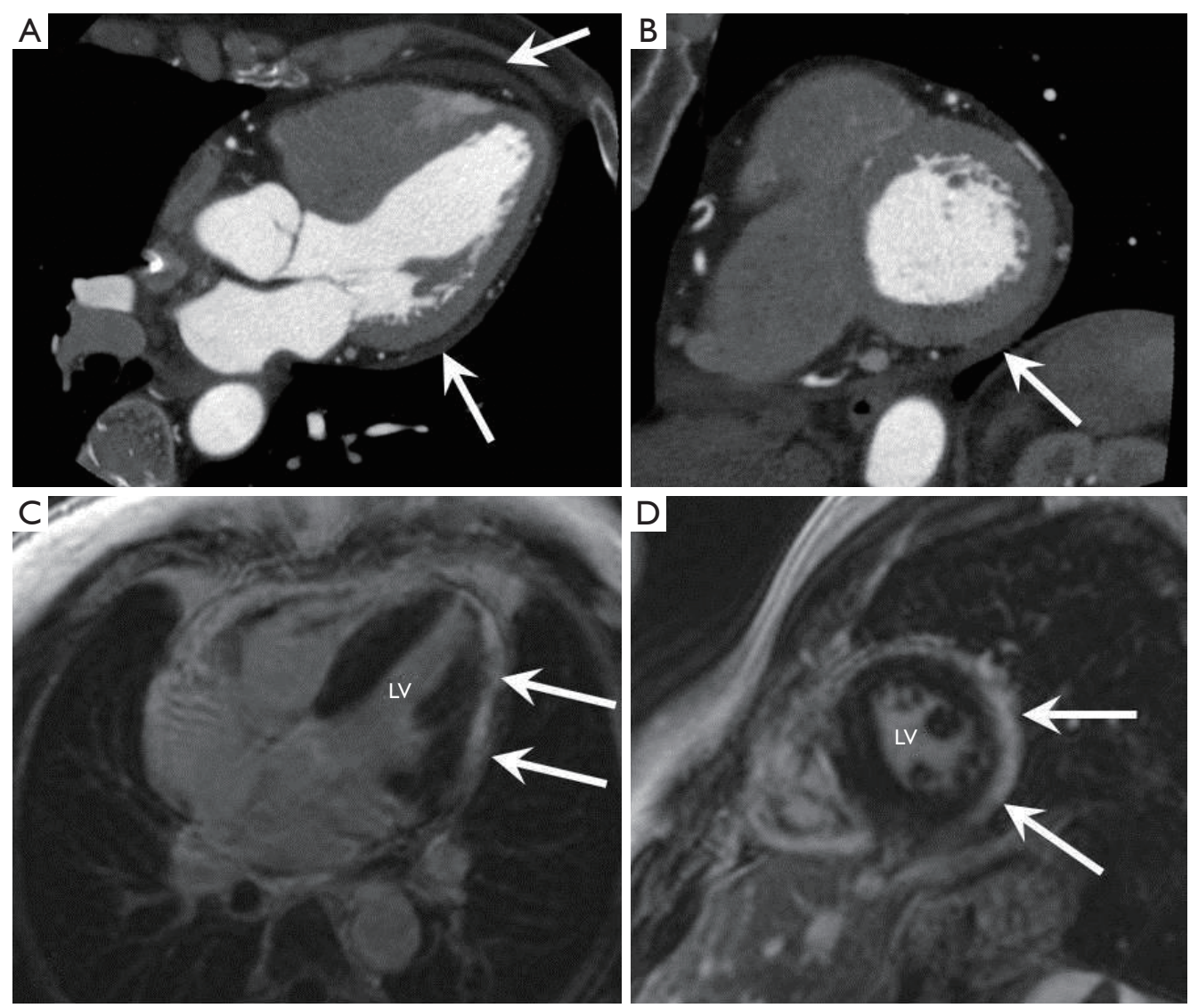

Figure 3 Acute pericarditis assessed with CT and MRI. (A,B) 3-chamber and short axis cardiac CT images showing subtle pericardial thickening and a small effusion; (C,D) 4-chamber and short axis late phase MRI images post gadolinium showing extensive pericardial enhancement (arrows). CT, computed tomography; MRI, magnetic resonance imaging.

pericardium, which prevents normal diastolic ventricular expansion and can result in both systemic and pulmonary venous congestion (1). Common causes include prior cardiac surgery, thoracic irradiation and tuberculosis. Imaging plays a particularly important role in distinguishing this condition from restrictive cardiomyopathy (which can have a similar clinical presentation) as only pericarditis benefits from pericardial stripping (pericardiectomy).

With CP there is usually pericardial thickening ( $>4 \mathrm{~mm}$ ) which may be localised or diffuse (11). TTE has a low accuracy in detecting pericardial thickening but this is readily detected with both CT and MRI (1). CT is far superior for detecting calcification but it should be noted that pericardial calcification can occur without constriction and vice versa (3). With MRI, pericardial thickness is best measured on black-blood images because chemical shift artefact on SSFP images (which occurs when there is a coexistent effusion) can lead to overestimation of pericardial thickness (12). Other imaging hallmarks of CP include tubular-shaped ventricles, biatrial enlargement, a flattened or reverse curvature interventricular septum and signs of impaired diastolic filling of the RV such as distension of the right atrium (RA), inferior vena cava and hepatic veins (6). With cine SSFP MRI haemodynamic processes can be identified-namely septal bounce, abrupt sensation of diastolic filling and also respirophasic variation in septal excursion. The later sign is demonstrated using a "realtime" sequence with the patient instructed to take a deep inspiration during the acquisition (6). As the diaphragm descends and the chest wall expands flattening and/or reversal of septal curvature (caused by diminished pericardial compliance) is a marker of constrictive physiology (1) (Figure 4). MRI has a reported sensitivity and specificity of $88 \%$ and $100 \%$ respectively, for the diagnosis of CP and in many cases can avoid the need for invasive cardiac catheterisation which is the current gold standard method 

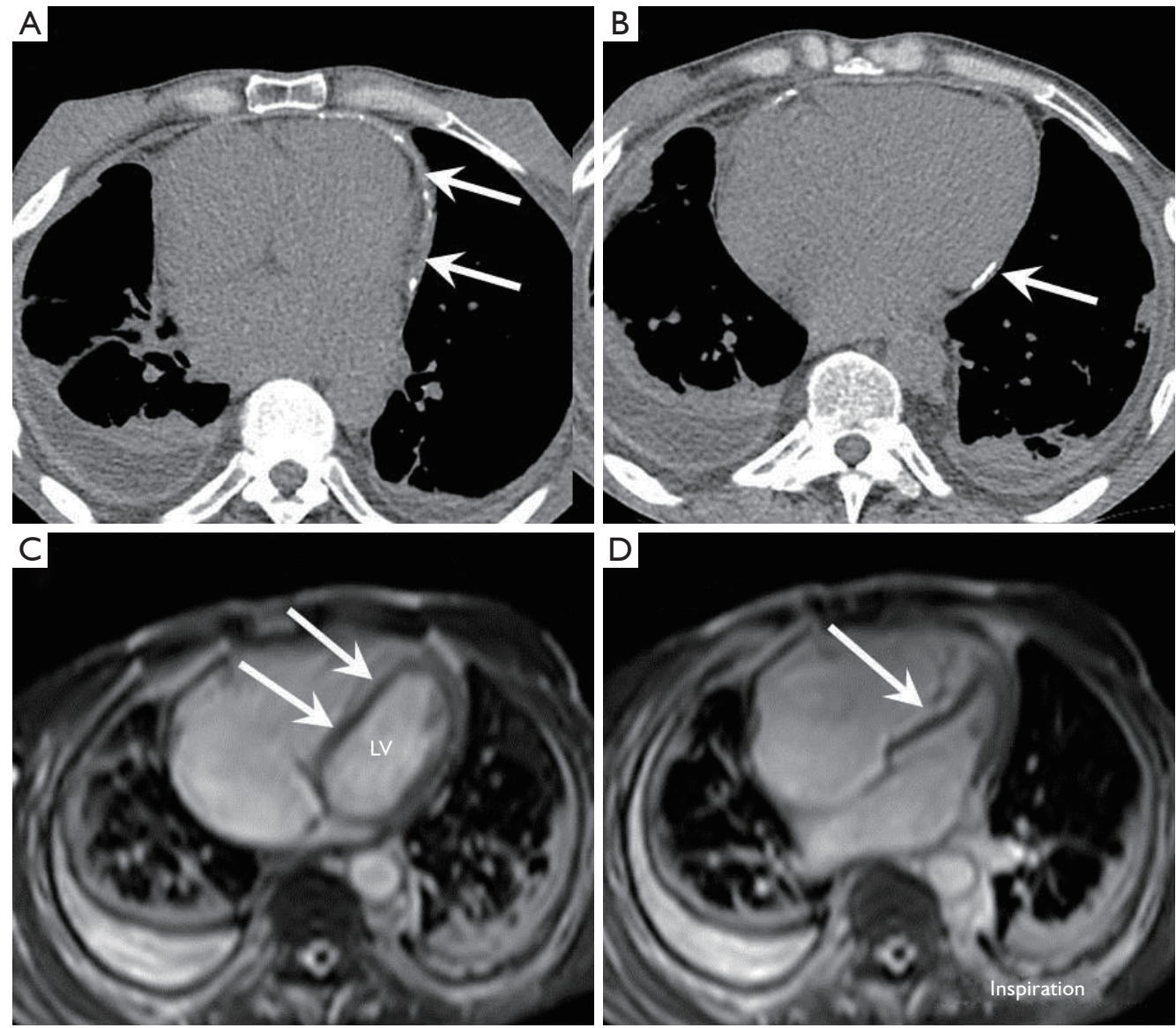

Figure 4 Constrictive pericarditis (CP). (A,B) Axial unenhanced CT images showing extensive pericardial calcifications and thickening (arrows); (C,D) real time cine MRI images showing respirophasic changes in septal morphology during inspiration which causes the septum to abruptly deviate due to diminished pericardial compliance as a marker of constrictive physiology (arrows). CT, computed tomography; MRI, magnetic resonance imaging.

of diagnosis (by showing dynamic changes in ventricular pressure curves during respiration) (6).

In patients with a confirmed diagnosis of $\mathrm{CP}$ who are being worked up for pericardiectomy cardiac CT is the technique of choice to aid with pre-operative planning owing to its high sensitivity for assessing the location and extent of pericardial calcification. For example in patients shown to have circumferential or extensive posterolateral pericardial calcification bilateral thoracotomy may be the preferred approach. CT is also particularly helpful to exclude significant concomitant coronary artery disease hence avoiding the need for a catheter angiogram (12).

\section{Effusive CP}

Effusive CP is characterized by impaired diastolic filling due to a concomitant pericardial effusion and constriction by the visceral pericardium. Classically the constrictive haemodynamics persist in these patients despite resolution or drainage of pericardial fluid $(1,6,12)$.

It is thought to most commonly be associated with malignancy and radiation induced injury and is present in around $8 \%$ of patients presenting with cardiac tamponade (13). The diagnosis of effusive CP traditionally requires cardiac catheterisation which shows a failure of reduction in right atrial pressure following pericardiocentesis with a concomitant diastolic ventricular pressure dip $(12,13)$. Non-invasive imaging can be helpful to confirm complete removal of pericardial fluid and MRI with late gadolinium enhancement imaging has been postulated to provide important prognostic information by demonstrating any ongoing pericardial inflammation $(6,13)$. 

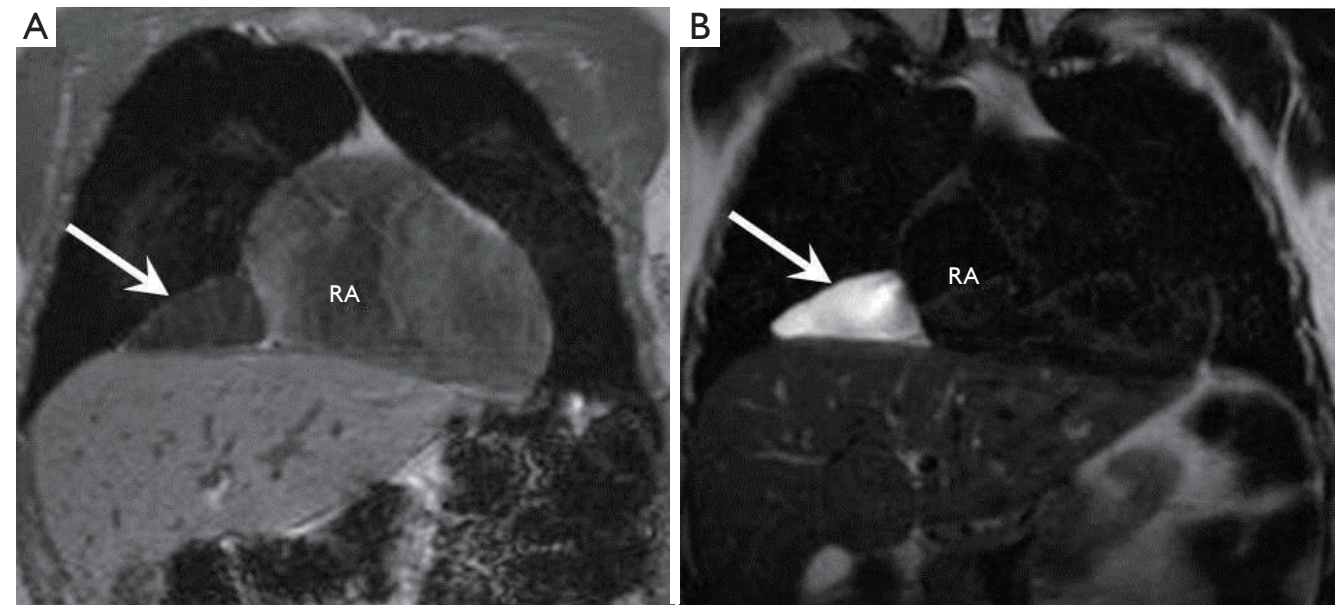

Figure 5 Pericardial cyst evaluated with MRI. (A) Coronal T1-weighted image showing a well-defined uniformly low signal intensity (fluid) lesion at the right cardiophrenic angle (arrow); (B) coronal T2-weighted image showing a uniformly high signal intensity (fluid) lesion. MRI, magnetic resonance imaging.

\section{Pericardial cyst}

Pericardial cysts are benign congenital lesions which arise from the fibrous pericardium and do not communicate with the pericardial space. The vast majority are found at the right cardiophrenic angle although they may occur anywhere in the mediastinum. They are unilocular and contain water based fluid without internal septa or solid components (4). Most remain asymptomatic and are found incidentally on imaging investigations performed for another reason although if large they may cause chest pain, cough and dyspnoea. A pericardial cyst typically appears as a thin-walled, unilocular nonenhancing lesion at the right cardiophrenic angle with near water attenuation values by CT. On MRI they show signs of water composition with uniform low signal intensity on T1- and high signal intensity on T2-weighted images (Figure 5). Occasionally a pericardial cyst may contain proteinaceous fluid and can exhibit high signal intensity on both T1- and T2-weighted imaging (12).

\section{Pericardial abscess}

Pericardial abscess is a walled off collection of pus within the pericardial space and most frequently occurs secondary to tuberculous pericarditis. Characteristic cross sectional imaging appearances are that of a localized biconvex collection within the pericardial space compressing the adjacent cardiac chambers (12). CT shows a variable wall thickness with peripheral enhancement with or without inner septations (Figure 6). On MRI the core may be better demonstrated appearing isointense to hyperintense on both T1- and T2-weighted images. T2-hypointensity of the core although rare, may be a specific marker for tubercular causes (6).

\section{Pericardial tumours}

Pericardial metastases are by far the most common malignancy to involve the pericardium. Primary tumours of the pericardium are extremely rare with mesothelioma being the most frequent followed by synovial sarcoma. Other tumours which can on occasion occur in the pericardium include lipomas, hemangioma, dermoids and teratomas. Although echocardiography may be used to screen for malignant involvement of the pericardium it rarely permits complete evaluation of a para-cardiac mass owing to limitations of acoustic windows. CT or MRI are generally required for accurate delineation of tumour size, extent and tissue characterisation-the presence of a haemorrhagic pericardial effusion, local invasion into the epicardial fat, myocardium, or into a cardiac chamber are typical imaging characteristics of an aggressive lesion.

\section{Pericardial metastasis}

Pericardial metastases are 100-1,000 times more frequent than a primary pericardial neoplasm and usually occur late 

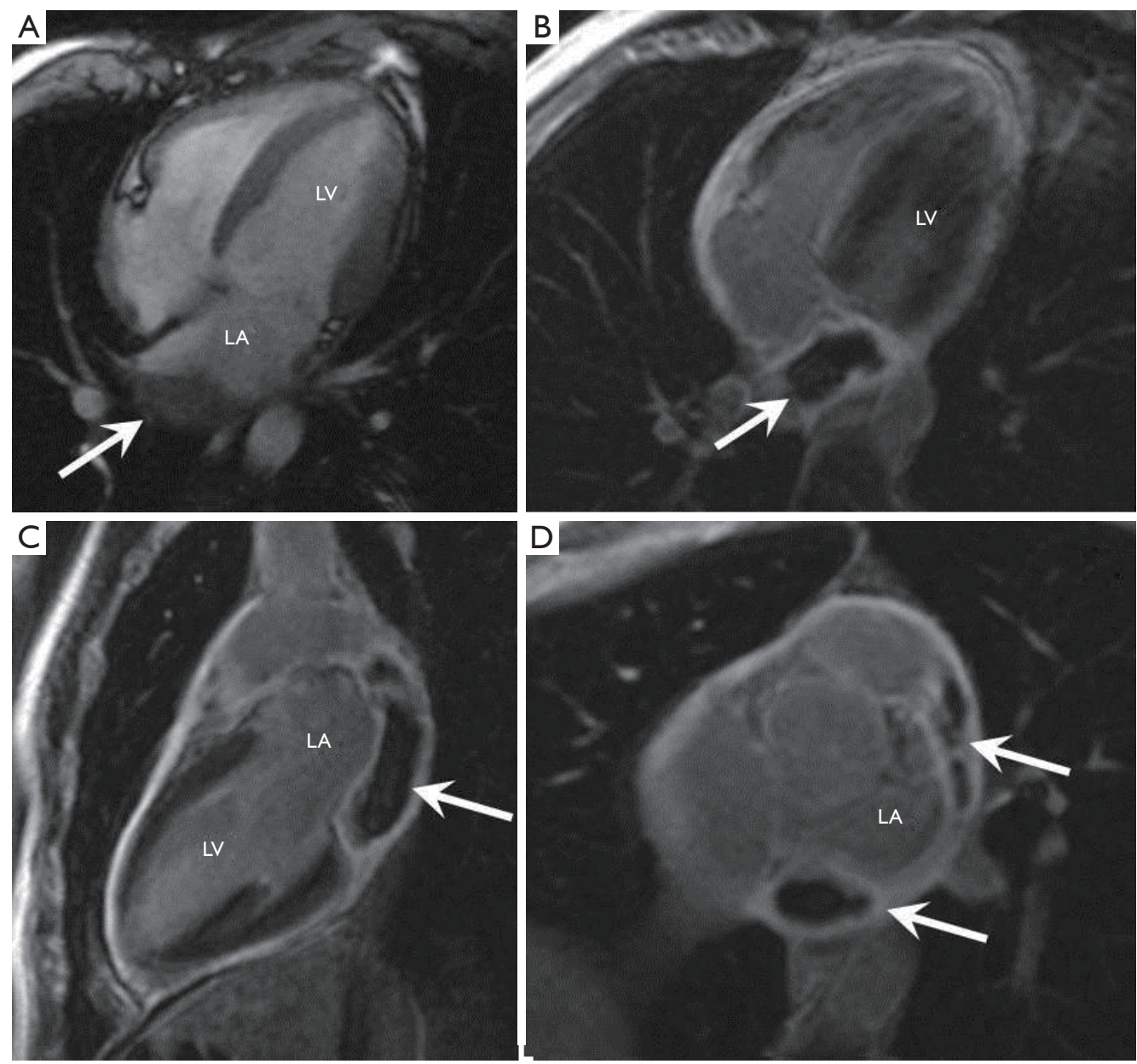

Figure 6 Tuberculous pericardial abscesses evaluated with MRI. (A) 4-chamber SSFP image showing an intermediate signal mass abutting the posterior wall of the left atrium (LA) (arrow); (B-D) late phase images following gadolinium showing avid peripheral enhancement and central dark fluid signal in keeping with pericardial abscesses (arrows). MRI, magnetic resonance imaging; SSFP, steady state free precession.

in the disease process. The most common sources are from breast, lung and renal primaries. Mechanisms of spread include direct extension, haematogenous and via lymphatic pathways (14). In patients with pericardial metastases cardiac tamponade is the most frequent cause of death.

Imaging appearances are generally non-specific but invariably comprise a large pericardial effusion and one or more soft tissue density nodules with variable degrees of contrast enhancement depending upon the primary site (Figure 7). On MRI most neoplasms exhibit low-signal intensity on T1- and high-signal intensity on T2-weighted images except for melanoma which exhibits high T1-signal intensity due to the unique T1 shortening properties of melanin (15).

\section{Pericardial mesotbelioma}

Pericardial mesothelioma is a malignant neoplasm arising from the mesothelial cells of the pericardium and accounts for $<1 \%$ of all thoracic mesotheliomas (14). It does not include primary pleural mesotheliomas which secondarily invade the pericardium. A definite causal relationship with asbestos exposure has not yet been established due to the small numbers of reported cases. The clinical presentation may mimic pericarditis with chest pain and dyspnoea secondary to progressive pericardial infiltration, encasement and/or tamponade. As with primary pleural mesothelioma the prognosis is poor and treatment is usually palliative with few 

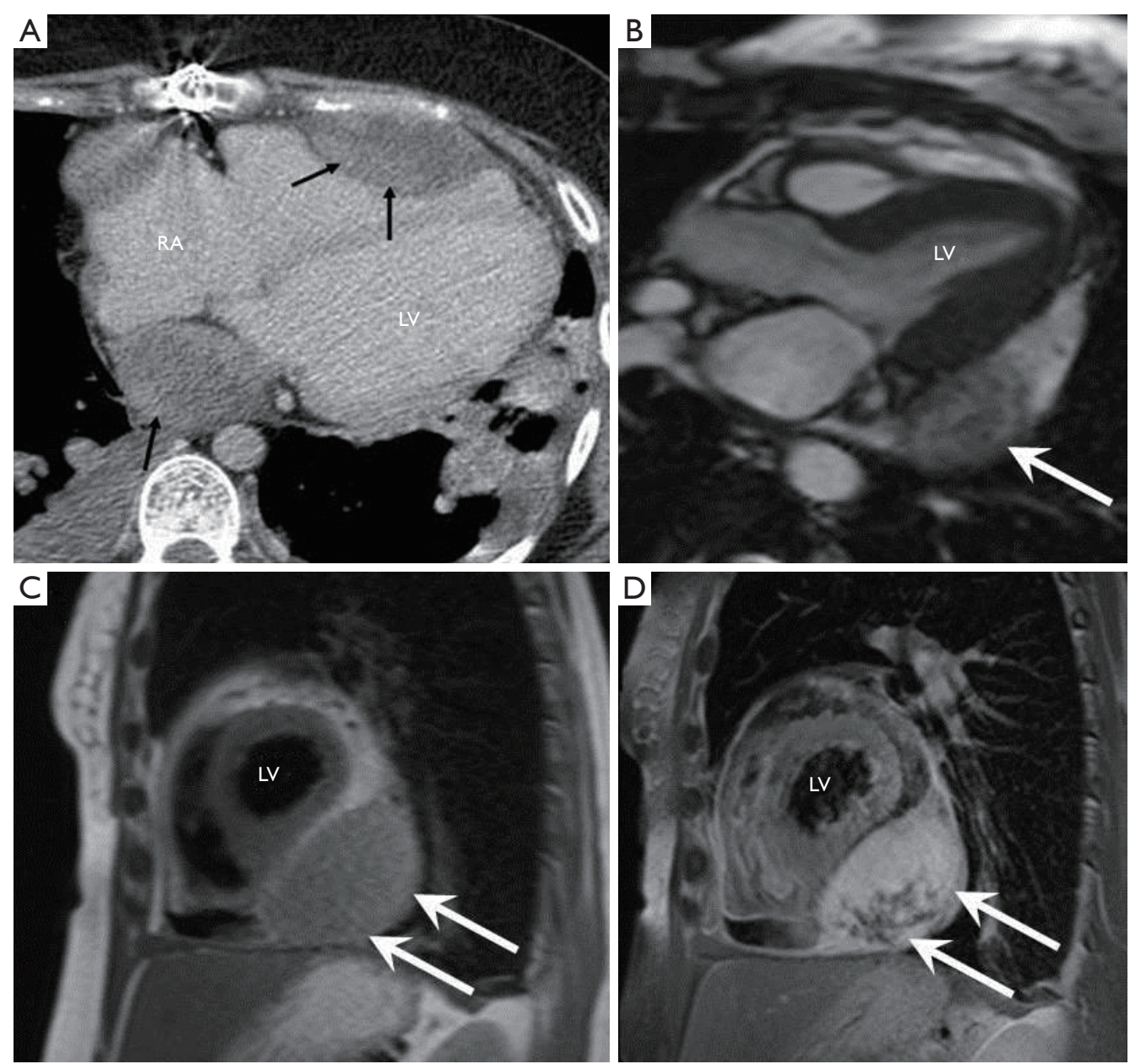

Figure 7 Pericardial metastases from breast cancer. (A) Axial venous phase (70 seconds) CT image showing multiple enhancing pericardial based nodules (arrows); (B) 3-chamber SSFP MRI image showing a large pericardial deposit with associated effusion adjacent to the lateral wall of the left ventricle (arrow); (C) short axis T1-weighted MRI image showing a pericardial deposit of intermediate signal intensity (arrows); (D) late phase image post gadolinium administration showing avid enhancement with central necrosis (arrows). CT, computed tomography; MRI, magnetic resonance imaging; SSFP, steady state free precession.

patients surviving more than 12 -months following initial diagnosis (15).

CT and MRI demonstrate multiple enhancing and coalescing pericardial masses usually with a large associated exudative effusion (Figure 8). Tumours are isointense to myocardial on T1-weighted imaging and heterogeneous on T2-weighted imaging (15).

\section{Pericardial synovial sarcoma}

Pericardial synovial sarcoma is an extremely aggressive tumour composed of spindle cells and epithelioid elements.
Prognosis is dire even with aggressive surgical de-bulking and chemotherapy. Imaging appearances are those of a large heterogeneous broad based mass, often centred on the right atrial free wall and with extensive invasion into surrounding structures (16). Signal intensity on MRI is heterogeneous but predominantly isointense to myocardium on T1-weighted and hyperintense on T2-weighted images reflecting areas of tumour tissue, necrosis and haemorrhage. SSFP signal is predominantly hyperintense with areas of high and low signal intensity corresponding to haemorrhage and necrosis respectively (Figure 9). Enhancement is typically avid (16). 

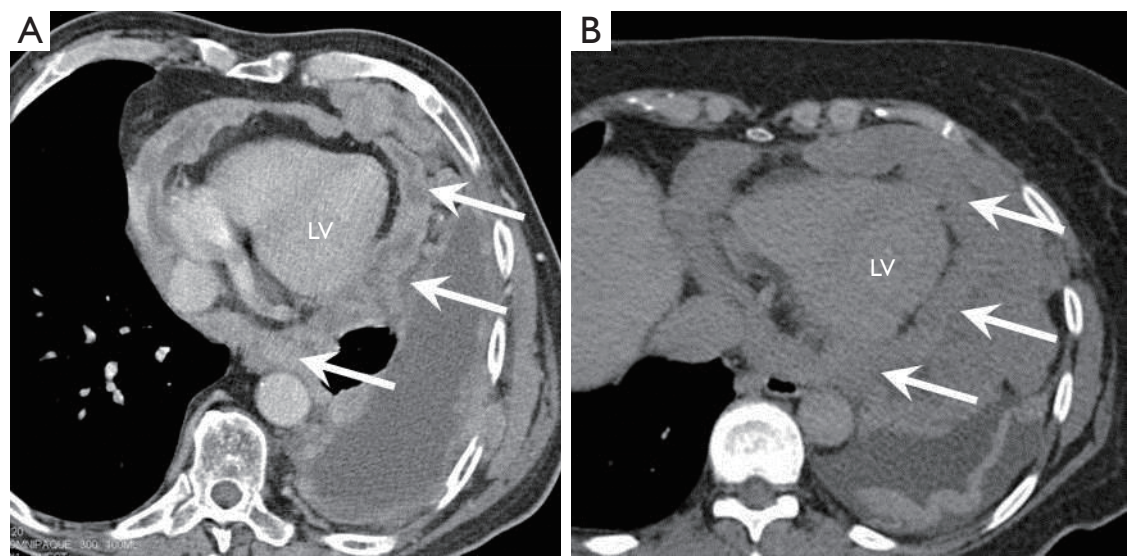

Figure 8 Primary pericardial mesothelioma assessed with CT. (A) venous phase axial image showing lobulated circumferential pericardial infiltration (arrows) with encasement of the heat and a large left sided pleural effusion with pleural nodules; (B) late phase axial image showing delayed washout of contrast medium from the malignant tissue (arrows). LV, left atrium; CT, computed tomography.
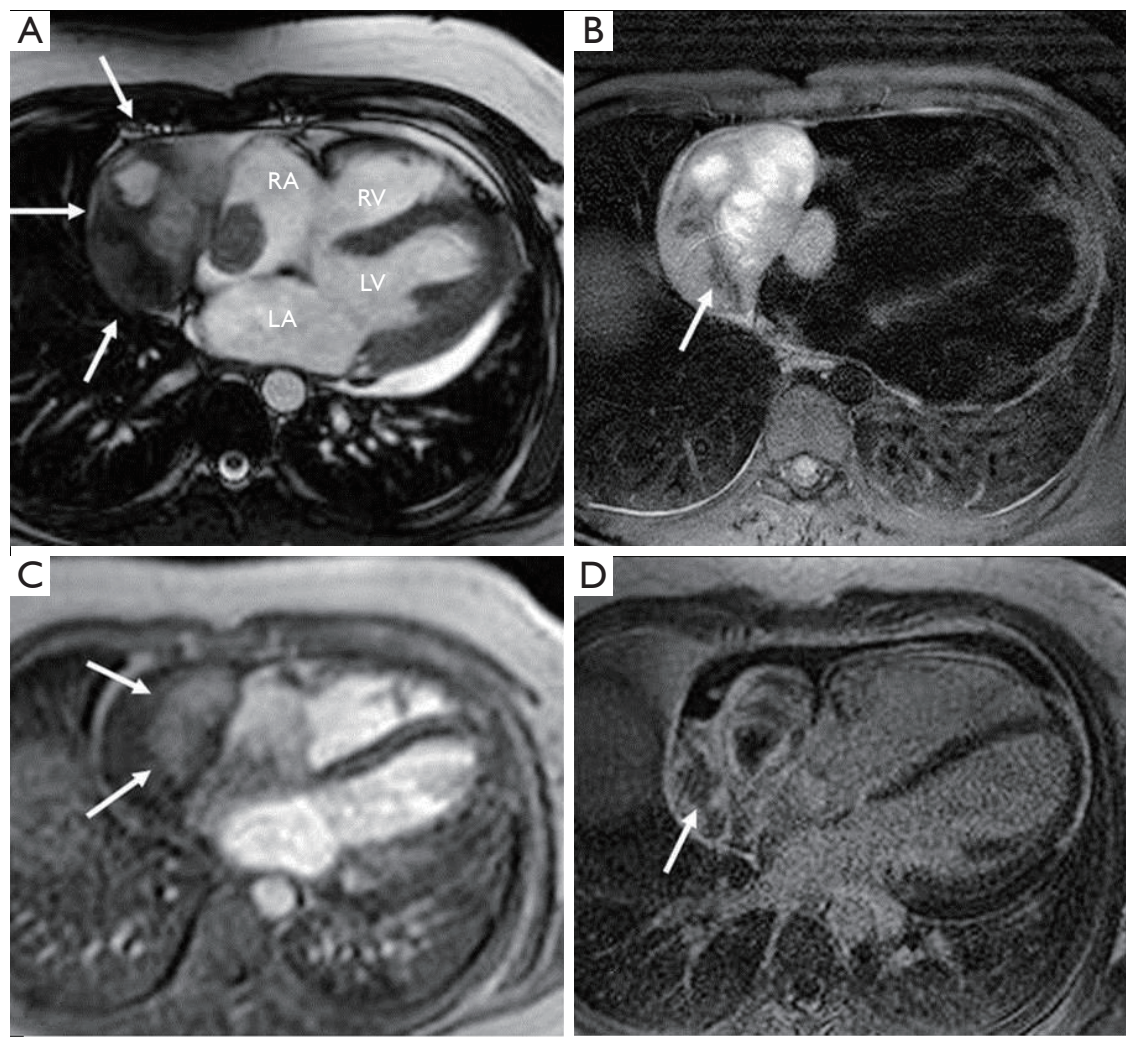

Figure 9 Pericardial synovial sarcoma assessed with multi-parametric MRI. (A) 4-chamber SSFP image showing a large heterogeneous signal mass adjacent to the right atrial free wall; (B) T2-weighted image showing the mass to contain areas of both solid and cystic elements. Low signal intensity centrally is in keeping with blood products (arrow); (C) first pass perfusion study showing avid enhancement of the central solid elements (arrows); (D) late phase image showing patchy residual enhancing tissue and non-enhancing (necrotic) elements (arrow). LV, left ventricle; LA, left atrium; RV, right ventricle; RA, right atrium; MRI, magnetic resonance imaging; SSFP, steady state free precession. 


\section{Conclusions}

CT and MRI are increasing used as complimentary imaging techniques to echocardiography for the assessment of pericardial diseases and can provide valuable additional clinical information. This paper has highlighted the role of multi-modality imaging across a range of pericardial disease processes. Cardiac imaging specialists should be familiar with the indications and clinical utility of these methods in order to direct an appropriate imaging strategy on a case by case basis.

\section{Acknowledgements}

None.

\section{Footnote}

Conflicts of Interest: The authors have no conflicts of interest to declare.

\section{References}

1. Yared K, Baggish AL, Picard MH, Hoffmann U, Hung J. Multimodality imaging of pericardial diseases. JACC Cardiovasc Imaging 2010;3:650-60.

2. Spodick DH. Macrophysiology, microphysiology, and anatomy of the pericardium: a synopsis. Am Heart J 1992;124:1046-51.

3. Peebles CR, Shambrook JS, Harden SP. Pericardial disease--anatomy and function. Br J Radiol 2011;84 Spec No 3:S324-37.

4. Kim JS, Kim HH, Yoon Y. Imaging of pericardial diseases. Clin Radiol 2007;62:626-31.

Cite this article as: Hoey ET, Shahid M, Watkin RW. Computed tomography and magnetic resonance imaging evaluation of pericardial disease. Quant Imaging Med Surg 2016;6(3):274-284. doi: 10.21037/qims.2016.01.03
5. Bull RK, Edwards PD, Dixon AK. CT dimensions of the normal pericardium. Br J Radiol 1998;71:923-5.

6. Hoey ET, Gulati GS, Ganeshan A, Watkin RW, Simpson $\mathrm{H}$, Sharma S. Cardiovascular MRI for assessment of infectious and inflammatory conditions of the heart. AJR Am J Roentgenol 2011;197:103-12.

7. Shah AB, Kronzon I. Congenital defects of the pericardium: a review. Eur Heart J Cardiovasc Imaging 2015;16:821-7.

8. Steinberg C, Pelletier MJ, Perron J, Kumar A, Champagne J. Sudden cardiac arrest due to subtotal absence of leftsided pericardium--case report and review of the literature. Congenit Heart Dis 2013;8:E92-8.

9. Hoey ET, Yap KS, Darby MJ, Mankad K, Puppala S, Sivananthan MU. Complete left pericardial defect: evaluation with supine and decubitus dual source CT. J Cardiovasc Comput Tomogr 2009;3:417-9.

10. Prakash P, Kalra MK, Stone JR, Shepard JA, Digumarthy SR. Imaging findings of pericardial metastasis on chest computed tomography. J Comput Assist Tomogr 2010;34:554-8.

11. Rajiah P, Kanne JP. Computed tomography of the pericardium and pericardial disease. J Cardiovasc Comput Tomogr 2010;4:3-18.

12. Bogaert J, Francone M. Pericardial disease: value of CT and MR imaging. Radiology 2013;267:340-56.

13. Syed FF, Ntsekhe M, Mayosi BM, Oh JK. Effusiveconstrictive pericarditis. Heart Fail Rev 2013;18:277-87.

14. Hancock EW. Neoplastic pericardial disease. Cardiol Clin 1990;8:673-82.

15. Burke A, Jeudy J Jr, Virmani R. Cardiac tumours: an update: Cardiac tumours. Heart 2008;94:117-23.

16. Sparrow PJ, Kurian JB, Jones TR, Sivananthan MU. MR imaging of cardiac tumors. Radiographics 2005;25:1255-76. 